\title{
Aperçus d'une genèse bilingue chez Jean-Joseph Rabearivelo
}

\section{Claire Riffard}

\section{(2) OpenEdition}

1 Journals

\section{Édition électronique}

URL : http://journals.openedition.org/genesis/2671

DOI : 10.4000/genesis.2671

ISSN : 2268-1590

\section{Éditeur :}

Presses universitaires de Paris Sorbonne (PUPS), Société internationale de génétique artistique littéraire et scientifique (SIGALES)

\section{Édition imprimée}

Date de publication : 4 juin 2018

Pagination : 81-92

ISBN : 979-10-231-0604-6

ISSN : 1167-5101

\section{Référence électronique}

Claire Riffard, «Aperçus d'une genèse bilingue chez Jean-Joseph Rabearivelo », Genesis [En ligne], 46 | 2018, mis en ligne le 01 juin 2019, consulté le 07 septembre 2019. URL : http:// journals.openedition.org/genesis/2671; DOI : 10.4000/genesis.2671 


\title{
Aperçus d'une genèse bilingue chez Jean-Joseph Rabearivelo
}

\author{
Claire Riffard
}

L'édition des brouillons bilingues du poète malgache Jean-Joseph Rabearivelo (1903-1937), procurée en 2012 par l'équipe «Manuscrits francophones» de l'ITEM 1 , a suscité la curiosité dans deux champs critiques : celui de la traductologie, désireuse de renouveler ses corpus de travail, et celui des études francophones, qui s'ouvrent aujourd'hui largement aux questions de plurilinguisme. L'analyse génétique d'un tel corpus permet aujourd'hui de faire avancer une réflexion commune qui a trait, au-delà d'un contexte géographique et historique singulier (Madagascar dans les années 1930), à la question complexe de la genèse en langues ${ }^{2}$ des littératures francophones.

\section{Les langues de Rabearivelo}

Maurice Martin du Gard, qui fait la connaissance du jeune écrivain à l'occasion d'un voyage à Madagascar en 1933, esquisse un portrait bien dans le ton de l'époque :

C'est vous dire si j'ai du goût pour Rabearivelo qui, pour rien au monde, n'abandonnerait le culte des ancêtres et en fit le fond même de ses plus beaux vers écrits dans un français très musical et très pur. Rien n'est doux comme de l'entendre fiancer à sa langue adoptive l'esprit de ses aïeux et de sa terre endormie. Les épaules étroites et timides, un vaste front de cuivre sous une chevelure terriblement noire et ébouriffée, c'est l'amoureux le plus jaloux de l'Afrique à la fois, de l'Inde et de 1'Europe 3 .

Martin du Gard insiste avec raison sur la complexité des représentations identitaires de Rabearivelo, sur sa volonté de syncrétisme entre plusieurs mondes dans un contexte de violence coloniale aiguë. Dans cette colonie, française depuis 1896, le français a statut de langue officielle sur fond de plurilinguisme 4 (variantes régionales du malgache, langues européennes importées au XIX ${ }^{\mathrm{e}}$ siècle par les missionnaires anglais, norvégiens...). Cette situation a très fortement fragilisé la langue malgache :

Genesis 46, 2018
Parallèlement à la francisation s'est s'instaurée une neutralisation officielle, mais non dans les faits bien sûr, de la langue malgache qui n'avait plus aucun statut légal : ni langue officielle, ni langue d'enseignement, ni langue nationale malgré l'unité linguistique évidente que l'on peut dégager grâce à la comparaison des différents régiolectes existants. Le malgache était, en définitive, relégué au rang de simple langue vernaculaire, et encore ${ }^{5}$ !

L'apprentissage scolaire de Rabearivelo se fait donc en français. Il fréquente l'école catholique jusqu'à ses 13 ans, puis prolonge sa formation initiale par des lectures personnelles assidues. Comme le résume vigoureusement Martin du Gard : «Il demandait du Lamartine aux Pères, on lui donna du Pierre L'Ermite, il se procura Baudelaire6. » À ses débuts poétiques en $1921^{7}$, sa maîtrise de la langue française est encore fragile. Il est néanmoins repéré par certaines figures du milieu littéraire colonial qui lui donnent libre accès à leurs bibliothèques; il se lance alors dans une

1. <http://www.item.ens.fr/francophone> (consulté en décembre 2017); Jean-Joseph Rabearivelo, Euvres complètes, Tome 2, Le poète, le narrateur, le dramaturge, le critique, le passeur de langues, l'historien. Coord. Serge Meitinger, Liliane Ramarosoa, Laurence Ink et Claire Riffard, Paris, CNRS Éditions, 2012.

2. Voir Olga Anokhina et François Rastier (dir.), Écrire en langues : littératures et plurilinguisme, Paris, Édition des archives contemporaines, coll. «Multilinguisme, traduction, création», 2015.

3. Maurice Martin du Gard, «Le voyage à Madagascar», Les Nouvelles littéraires, 2 décembre 1933.

4. Voir Stéphanie Benson, Les Aventuriers de la langue fourchue : l'écriture multilingue et la désintégration de l'espace colonial, thèse de doctorat, université de Bordeaux III, 2011.

5. Élisa Rafitoson, «Bilingue dans un milieu de diglossie», Notre Librairie, $\mathrm{n}^{\circ} 110$, septembre 1992, p. 46.

6. Maurice Martin du Gard, «Le voyage à Madagascar», Les Nouvelles littéraires, 2 décembre 1933.

7. Jean-Joseph Rabearivelo, Le couchant, 24 mai 1921. Éditeur : Projet e-Man, Claire Riffard, Institut des textes et manuscrits modernes, CNRS-ENS. Consulté le 23 mars 2017 sur la plate-forme e-Man : <http://eman-archives.org/francophone/items/show/2491> (consulté en décembre 2017). 
frénésie de lectures, guettant l'arrivée des dernières livraisons de revues avant-gardistes et passant commande des succès du temps aux libraires parisiens. Trois années plus tard, à l'âge de 21 ans, Rabearivelo est à même de publier un premier recueil : La Coupe de cendres $^{8}$, qui le consacre poète en langue française. Ce premier succès est salué par le romancier Pierre Mille dans un discours prononcé en 1926 devant l'Académie des sciences coloniales : «Admirez avec moi ce miracle, Messieurs [...]. C'est en français, avec des rimes françaises, qu'un Malgache exprime aujourd'hui ses élans, ses aspirations, ses rêves ${ }^{9}$ ».

Rabearivelo mène parallèlement une activité de poète, de dramaturge et de théoricien de langue malgache. On a peine à se figurer ce que cet acte comportait de volontarisme en ces temps coloniaux. La maîtrise des subtilités de la langue et des codes littéraires traditionnels (art du discours, joutes oratoires, opéra paysan...) nécessitait un engagement intellectuel considérable, tant cette littérature avait été mise sous le boisseau depuis les débuts de l'occupation française ${ }^{10}$. Si Rabearivelo a été salué pour l'excellence de son français, comme nous l'avons vu, sa maitrise de la langue malgache se révèle progressivement tout aussi exceptionnelle : «Il a écrit des poèmes malgaches qui n'ont rien à envier à ceux qu'il a écrits en langue étrangère, et qui ont paru dans de nombreux journaux et revues : Ny Mpandinika, Ranovelona, Kalokalo

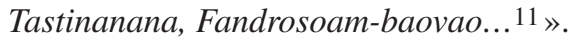

Le jeune écrivain est donc l'héritier d'une double tradition littéraire, qu'il cherche à préserver en ses deux versants. Il crée dans tous les genres (théâtre, romans, nouvelles, poésie, essais, y compris historiques) dans ses deux langues d'écriture, mais ne parvient à faire publier qu'une part minoritaire de sa production : plusieurs recueils de poèmes en français, dont Presque-Songes (1934) et Traduit de la nuit (1935), révélés au monde des lettres par L. S. Senghor dans son Anthologie de la nouvelle poésie nègre et malgache de 1948, et en malgache essentiellement des pièces de théâtre et des essais ${ }^{12}$.

\section{Le brouillon bilingue du recueil Presque-Songes}

À l'été 2008, un groupe de recherche constitué de spécialistes français et malgaches sollicite des enfants de Rabearivelo l'accès aux archives pour envisager une étude génétique de l'œuvre. La famille se montre particulièrement favorable à cette proposition et ouvre les portes de la demeure, située dans la périphérie rizicole d'Antananarivo, où vit encore l'une des filles de l'écrivain, Noro Rabearivelo-Rakotomanga. Au premier étage, mêlé à des albums d'entomologiste et des archives administratives, est conservé un ensemble de cahiers d'écolier et de feuillets volants qui se révèle un premier pan de l'archive littéraire du poète, vieille de 80 ans déjà. Les recherches successives permettront de localiser ailleurs dans la maison deux malles d'archives qui contiennent un ensemble d'au moins 5000 feuillets. Une partie de ces documents est relative à des textes inédits (nouvelles, essais historiques ou littéraires, pièces de théâtre..., ainsi qu'un journal intime de 900 feuillets entièrement rédigé en français, les Calepins bleus ${ }^{13}$ ). L'exploration révèle également un stupéfiant petit cahier, renfermant le brouillon entièrement bilingue du recueil poétique Presque-Songes, qui va nous occuper ici.

Ce document se présente sous la forme d'un cahier d'écolier de 50 pages format A5, rédigé recto-verso, en mauvais état de conservation. La disposition des poèmes sur la page est toujours identique : la version malgache figure à gauche, la version française à droite. Le trait central séparant les deux versions a été tracé avant l'écriture dans le cas des trois premiers poèmes de Presque-Songes ${ }^{14}$; dans les poèmes suivants, il vient après et suit en règle générale le tracé de la version malgache.

8. Jean-Joseph Rabearivelo, La Coupe de cendres, Tananarive, G. Pitot de la Beaujardière, 1924.

9. Cité par Locha Mateso, dans La Littérature africaine et sa critique, Paris, ACCT-Karthala, 1986, p. 91. Rabearivelo venait de publier des poèmes dans la revue Les Nouvelles littéraires.

10. C'est par les colons eux-mêmes qu'elle commence à être remise au goût du jour au début du XXe siècle. Une académie malgache est créée par le général Gallieni en 1902, dont l'un des axes porte sur une fixation de la langue; Rabearivelo y est reçu en 1932 en qualité de correspondant.

11. Siméon Rajaona, manuel de littérature malgache Takelaka Nostongaina, Antananarivo, Imprimerie nationale, 1961. Notre traduction.

12. Pour la seule année 1926 : «Fa velona indray», dans Ny Ranovelona $\left(\mathrm{n}^{\circ}\right.$ 10, janvier 1926), «Eritreritra fahavaratra», dans Ny Mpandinika (19-01-1926), «Tsy Embona» (Ny Mpandinika, $\left.{ }^{\circ} 125,16-04-1926\right)$, «Nosoratana tao anatin'ny fangorakorahana» (Ny Mpandinika, $\mathrm{n}^{\circ} 128$, 07-05-1926)...

13. Claire Riffard, «Bleu à l'âme, les Calepins bleus de J. J. Rabearivelo», La Faute à Rousseau, n 56, 2011, p. 55-56.

14. Le cahier de brouillon contient, à la suite des poèmes de PresqueSonges, ceux du recueil «jumeau» Traduit de la nuit (publié en 1935), également constitué de trente poèmes en français et en malgache. 
Ce document est probablement unique en son genre. Il atteste d'une écriture conjointe, quasi simultanée, d'une série de poèmes bilingues dont seule la version française sera éditée par l'écrivain en 193415. Si ce cahier était connu des quelques spécialistes de Rabearivelo, puisqu'il avait notamment servi en 1960 à établir la version malgache des poèmes pour l'édition posthume du recueil16, il n'avait été ni diffusé ni étudié. Il est maintenant consultable en libre accès sur la plateforme d'édition numérique EMAN de l'ITEM 17 .

J'ai eu l'occasion d'évoquer ailleurs ${ }^{18}$ les raisons qui ont pu amener Rabearivelo à ne publier ce recueil qu'en français, même s'il affirme à plusieurs reprises avoir l'intention, de publier, «plus tard», sa version malgache. Ces raisons sont pour partie politiques ${ }^{19}$ - elles touchent à la complexité d'une réception en contexte colonial -, et pour partie d'ordre intime. Je renvoie à ces vers du poème «Lamba» dans Presque-Songes, qui disent en vers à la fois le pragmatisme et l'idéal d'une relation à la langue française :

[...] dans cette langue que j'ai choisie

pour préserver mon nom de l'oubli,

dans cette langue qui parle à l'âme

alors que la nôtre murmure au cœur.

Mais Rabearivelo a-t-il vraiment choisi entre ses « deux amours / [S]on pays et Paris ${ }^{20}$ » ? Moradewum Adejunmobi résume la position prise par le poète en $1931:$ R. proudly dons his devotion to both Malagasy and French cultures. At the "hour of testing", he chooses both 21 ». Quand il fallut choisir entre ses deux langues d'écriture, Rabearivelo choisit les deux. Les manuscrits de travail de l'écrivain nous permettent d'approcher ce mouvement créatif bilingue des années 1931-1932.

\section{Écrire simultanément en deux langues, une utopie?}

La parole double s'avère une fois de plus impossible, et je me retrouve scindé en deux moitiés, aussi irréelles l'une que l'autre22.

Tzvetan TODOROV

«La parole double s'avère impossible», cette affirmation de Tzvetan Todorov se fonde sur une expérience personnelle mais éclaire également ses recherches fondatrices sur le bilinguisme et l'autotraduction. Beckett pointait déjà les «wastes and wilds of self-translation ${ }^{23}$ ». Pour tenter de saisir la spécificité d'un texte double en évacuant la question du texte de référence, les commentateurs ont pu utiliser des formules comme «textes parallèles » 24 ou «textes jumeaux » («twin textes») 25 . À la différence cependant de la pratique beckettienne qui donne naissance à deux textes inscrits dans une temporalité qui peut aller de quelques semaines à plusieurs années et qui représente un cas classique et exemplaire de ce qu'on appelle l'autotraduction, l'écriture bilingue de Rabearivelo dans Presque-Songes semble surgir simultanément. L'analyse minutieuse du brouillon rédactionnel bilingue (MS1.PS26), complétée par celle d'un dactylogramme préparant les épreuves de la version

15. Jean-Joseph Rabearivelo, Presque-Songes (poésie) [poèmes hova traduits par l'auteur], présentation de Robert Boudry, eaux-fortes d'Urbain-Faurec, Tananarive, Éditions Henri Vidalie, Librairie des Arts Graphiques, 34 x $30 \mathrm{~cm}$, non paginé (78 p.), 1934.

16. Jean-Joseph Rabearivelo, Presque-Songes, Tananarive, Les amis de Rabearivelo, 1960, 218 p.

17. <http://eman-archives.org/francophone/items/show/1855> (consulté en décembre 2017).

18. Claire Riffard, «Étude de manuscrits malgaches bilingues de J. J. Rabearivelo», dans Olga Anokhina (dir.), Multilinguisme et créativité littéraire, Louvain-La-Neuve/Paris, Academia/L'Harmattan, coll. «Au cœur des lettres», $n^{\circ} 20,2012$, p. 55-65 et p. 57.

19. Moradewum Adejunmobi rappelle un contexte politique assez tendu : «It seems likely that the almost tangible silence of Malagasy literature during and following the V.V.S affair was one of the factors that prompted Rabearivelo to seek to invest French Language with Malagasy values so as to keep alive the institution of Malagasy literature. » (Moradewum Adejunmobi, J. J Rabearivelo, literature and lingua franca in Colonial Madagascar, New York, Peter Lang, 1998, p. 54).

20. Pour reprendre le célèbre couplet de Joséphine Baker, strictement contemporain.

21. Moradewum Adejunmobi, op. cit., p. 106.

22. Tzvetan Todorov, «Bilinguisme, dialogisme et schizophrénie», dans Abdelkébir Khatibi (éd.), Du bilinguisme, Paris, Denoël, 1985, p. 11-38 et p. 23.

23. Voir Rainier Grutman, «Auto-translation», dans Routledge encyclopedia of translation studies, 1998, p. 17-20.

24. James Mc Guire, «Beckett, the Translator, and the Metapoem», World Literature Today, vol. LXIV, 2, printemps 1990, p. 260.

25 . Raymond Federman, A voice within a voice : Federman translating/ translating Federman, 1996 <www.federman.com/rfsrcr2.htm> (consulté en décembre 2017)

26. <http://eman-archives.org/francophone/items/show/1855> (consulté en décembre 2017). 
française (TP1.PS27), permet d'approfondir cette énigme d'une écriture conjointe.

\section{Notations bilingues en page de couverture}

Sur la page de couverture (fig. 1) du cahier d'écolier qui contient le brouillon rédactionnel, Rabearivelo hésite entre plusieurs propositions de titre, et travaille déjà en deux langues.

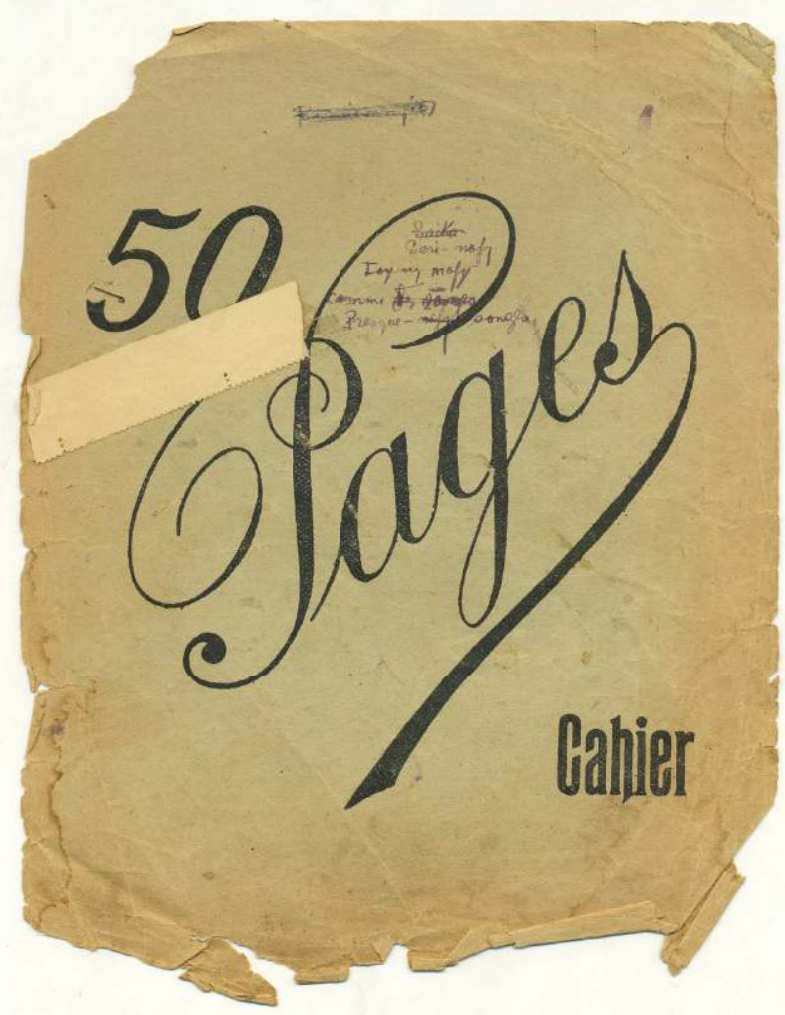

Fig 1 : J. J. Rabearivelo, détail de la page de couverture du brouillon rédactionnel de Presque-Songes, cahier d'écolier, format A5, archives privées famille Rabearivelo, Madagascar

$$
\begin{gathered}
\text { Saika- } \\
\text { Sari-nofy } \\
\text { Toy ny nofy } \\
\text { Comme des songes } \\
\text { Presque-nofy songes } 28
\end{gathered}
$$

Le titre a d'abord été porté en langue malgache. Le premier mot, biffé, «Saika-», est un préfixe qui a le sens de «sur le point de». Cette première esquisse est remplacée successivement par deux autres formules, «Sari-nofy» et «Toy ny nofy », où le substantif «nofy» [songe] est précédé de «sary » [image, ressemblance], puis de «toy» [comme]. L'hésitation se lit donc, dans les premières formulations en langue malgache, entre plusieurs mots qui signifient «presque» et actualisent chacun l'un des sens de cet adverbe polysémique en français. Rabearivelo formule ensuite en français l'équivalent de l'expression «Toy ny nofy»: «Comme des songes» avant d'envisager finalement un titre bilingue, «Presque-nofy», qui sera biffé à son tour pour aboutir à «Presque-songes » en français.

L'analyse de cette recherche de titre fait état d'un mouvement d'écriture allant verticalement du malgache vers le français; le parcours transformationnel part du malgache, passe par une construction entre deux (Presque-nofy) pour aboutir à un titre français qui porte - par sa structure atypique - la marque sémantique et syntaxique du malgache. Mais elle montre aussi la direction inverse et ouvre la voie à une genèse bilingue réciproque, puisqu'on peut également lire les premières lignes comme des tentatives de traduire du français vers le malgache les acceptions polysémiques de l'adverbe «presque», mentalement présent mais non encore couché sur le papier.

\section{Topographie bilingue}

Entrons maintenant dans la fabrique du texte, en analysant le brouillon rédactionnel du poème «Asara/Été », qui ouvre le recueil29. Il est daté au bas des deux versions du 22 juin 1931 (fig. 2).

Le chercheur se pose inévitablement la question : quelle version précède l'autre? D'un côté, le brouillon rédactionnel affiche une symétrie parfaite entre les deux versions : le trait central a été tracé préalablement à l'écriture (le poète avait donc un projet d'écriture bilingue) et la date identique portée au bas de chaque version proclame la contemporanéité de l'écriture. Inversement, l'inscription physique de l'écriture

27. <http://eman-archives.org/francophone/items/show/2124> (consulté en décembre 2017).

28. Les transcriptions sont celles de l'auteur de l'article.

29. Liminaire dans le brouillon rédactionnel, le poème figurera en troisième position dans l'édition originale française de 1934 (derrière «Lire » et «Le Poëme»). 


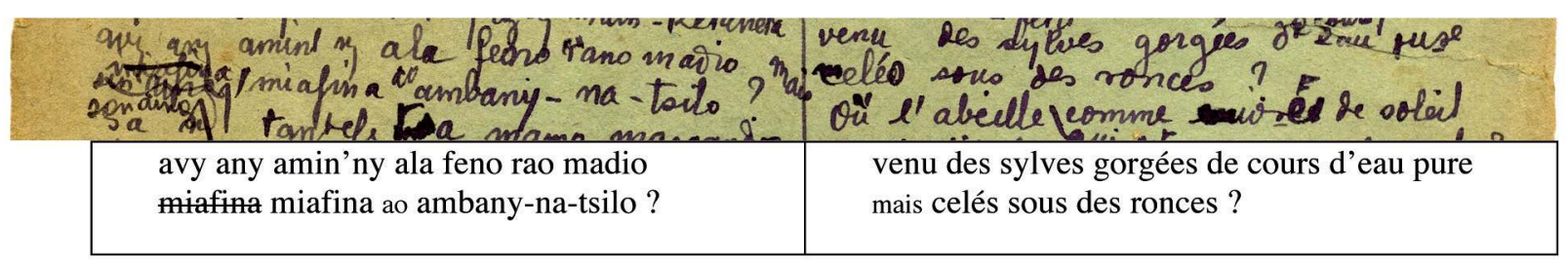

Fig. 5 : brouillon rédactionnel de Presque-Songes, v. 10 et 11 (détail)

en malgache sur la partie gauche de la page laisse penser qu'elle a été première, si l'on s'en tient au mouvement naturel de l'écriture (de la gauche vers la droite). Cependant, nous allons l'observer que ce mouvement est partiellement contredit par une étude génétique approfondie qui, pour certains vers, permet de supposer une antériorité de l'écriture en français. L'analyse détaillée du processus de création permet de mettre au jour un processus complexe où les deux versions du poème ne sont pas simplement successives mais interagissent dès la campagne d'écriture.

Le poème «Été» est exemplaire de cette recherche poétique, qui oscille en permanence entre un constat d' «irréduc-

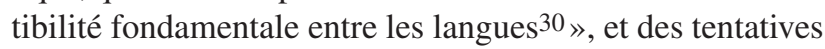
d'interaction.

\section{Deux univers irréductibles}

Rabearivelo est confronté à une différence irréductible entre deux univers culturels et deux systèmes linguistiques. Dans sa version malgache, le poème s'ancre dans la culture des Hauts plateaux, notamment à travers l'usage d'un lexique spécifique, et ce dès son titre. En effet, le substantif «asara» ne signifie pas à proprement parler «été», que l'on désigne communément par «fahavaratra» (littéralement «la saison de la foudre», d'où «saison des pluies, été»). «Asara» renvoie au premier mois du calendrier traditionnel, la «saison du fandroana » (du bain royal $)^{31}$; le mot porte donc un sens plus rituel, lié à la royauté malgache des Hautes terres. La date d'écriture inscrite au bas des deux versions - 22 juin 193132 - n'est signifiante que dans un calendrier français, où elle correspond au premier jour de l'été.

Ainsi, dès l'orée du poème, Rabearivelo se situe au confluent de deux traditions qu'il conjoint sur le papier.

Plus loin, aux vers 14 et 15, Rabearivelo use d'un réseau lexical qui renvoie aux gestes coutumiers observés à Madagascar lors d'un décès ${ }^{33}$, notamment ceux de l'esclave, «ankizivavy», qui va laver ses vêtements au bord du fleuve, cheveux dénoués. La connotation servile d' «ankizivavy » n'apparaît pas en français dans la traduction littérale «femme-enfant». Dans d'autres poèmes, Rabearivelo donnera comme correspondant malgache à l'expression française «femme-enfant» les substantifs «tovovavy» [jeune femme] ou «zazavavy» [jeune fille]. Dans le poème qui nous occupe, c'est le mot «ankizivavy» (qui signifie «adolescente ${ }^{34}$ », mais aussi «esclave (femme) ${ }^{35}$ ») qui est choisi. La profondeur symbolique de l'image n'est donc pas accessible dans la version française, qui n'évoque au lecteur qu'une banale scène de bain.

À l'inverse, Rabearivelo ne peut rendre en malgache la modulation de ton des vers 10 et 11 , qui confine à la préciosité (fig. 5).

Au vers 10, le substantif «sylves», sans doute inspiré au poète par ses lectures de Valéry et de Mallarmé, est plus communément rendu en malgache par «ala» [forêt]. Le vers a-t-il été écrit préalablement en français? Le jeu en langue française avec les références littéraires du temps, sous forme

30. Formule utilisée par Zeender Berset à propos de la poésie bilingue de Beat Christen; voir Muriel Zeender Berset, Écrire entre les langues, Slatkine, Genève, 2010, p. 253.

31. S. J. Malzac, Dictionnaire Malgache - Français, Fianarantsoa, Éditions Ambozontany, 1993 (1re édition 1888).

32. Rabearivelo se suicidera un 22 juin, en 1937.

33. «Le premier mercredi suivant l'enterrement, la famille du défunt est tenue de faire une grande lessive au bord d'une eau courante (celle-ci, par son mouvement, est censée emporter le malheur et empêcher son séjour continu auprès des vivants). En signe de deuil, les cheveux se portent épars. » (Ariane Andriamaharo, Écriture et création poétique dans l'œuvre de Rabearivelo, le poète des contradictions, thèse de doctorat, université de Paris XII, 1989, p. 117).

34. Narivelo Rajaonarimanana, Dictionnaire du malgache contemporain, Paris, Éditions Khartala, 1995.

35. S. J. Malzac, op. cit. 


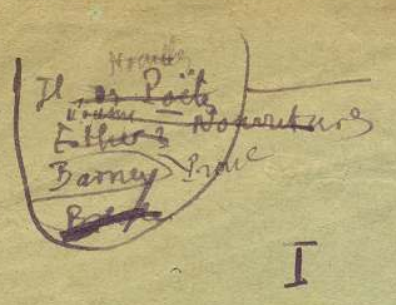

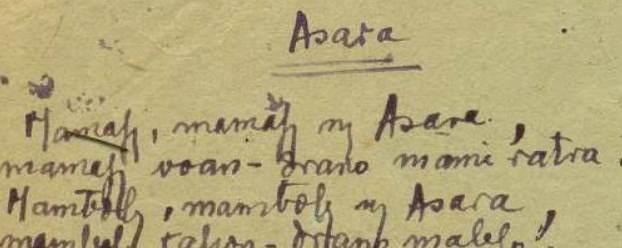

Mamaraf, manaf, mambol, mantiaf, Tham of manbolf anat sontamby.

Fory ary ino' no livi'in a salob?

- Moa un voron-Kel ? a main-Ketaher que amind $n$ ala feeno tano mavio A I miafin "ambani-na-toito?

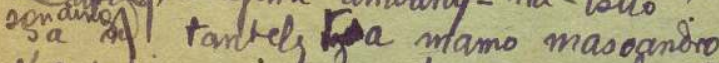
Ra lahim aininda anat sampaho? Sa ankivary vas mamala olo

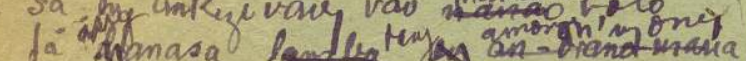
Sa bharano an ho an no vet a,

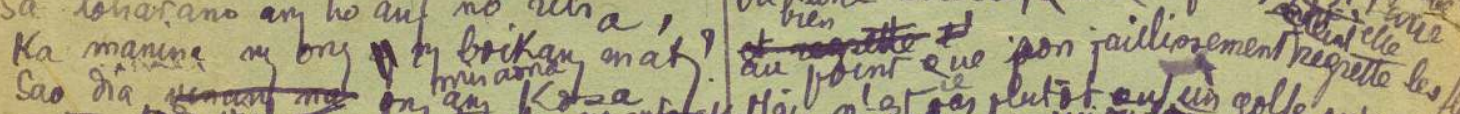

I Eré

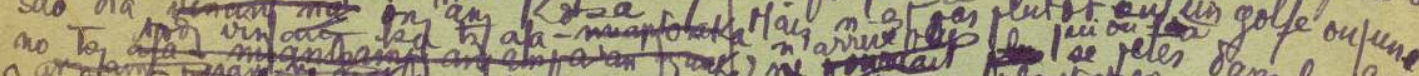

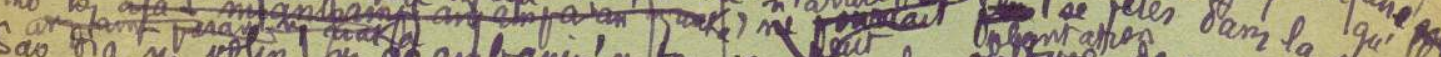

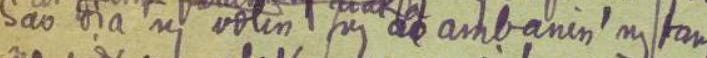

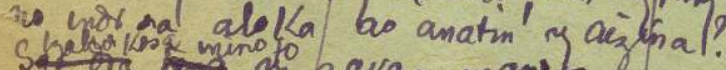

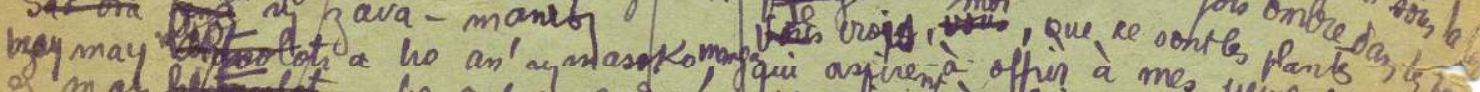

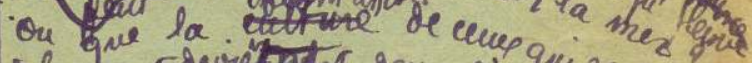

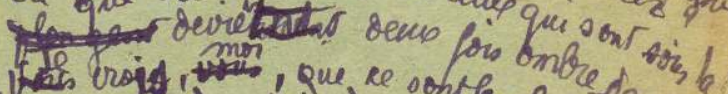

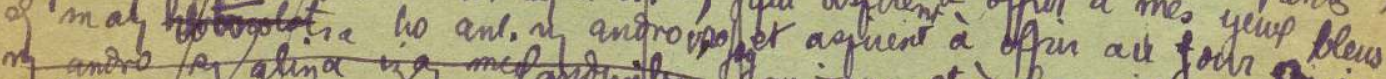

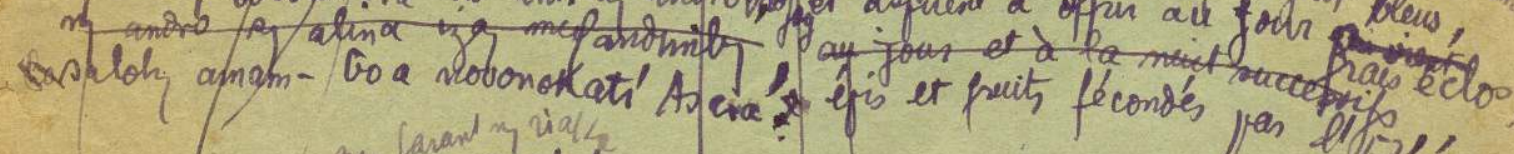

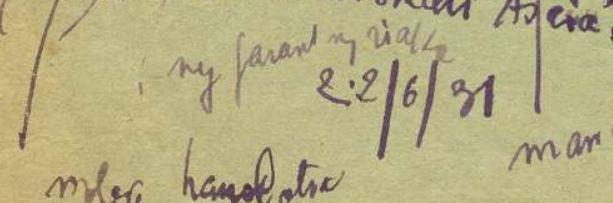
Plante, plante l'E hé plante veq tiges drear freleo. Seme, ieme t plante, ilante flante.

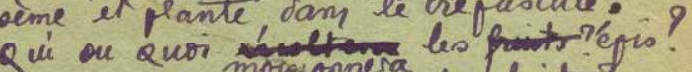

Es ce lo pa piet. vena des dybes gorgées jo sour puse

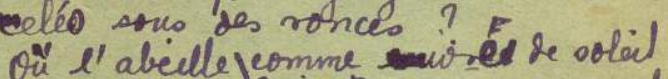
et qui titube qui cort cours os braschs? Or la femme-enfant pui irent re it acie we laven des effet an bow dus orepine source, quelate part, $z^{\prime} 35$, En

more handetio $17 / 2 / 6 / 3$

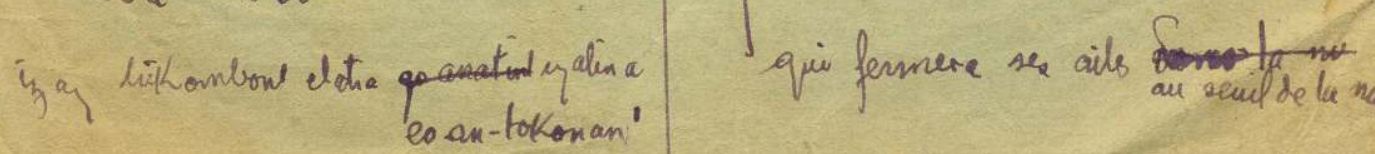
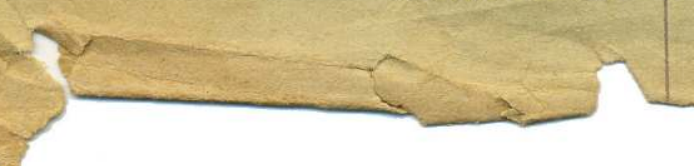

Fig 2 : Jean-Joseph Rabearivelo, brouillon rédactionnel de Presque-Songes, $\mathrm{f}^{\circ}$ 1v, cahier d'écolier, format A5, archives privées famille Rabearivelo, Madagascar 


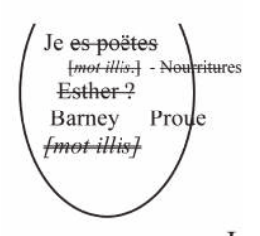

I

\begin{tabular}{|c|c|}
\hline Asara & Eté \\
\hline 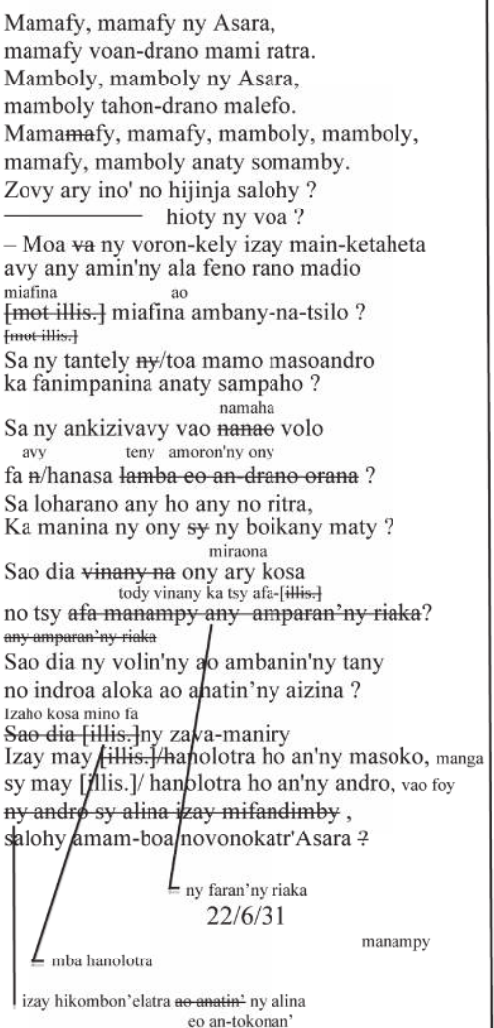 & 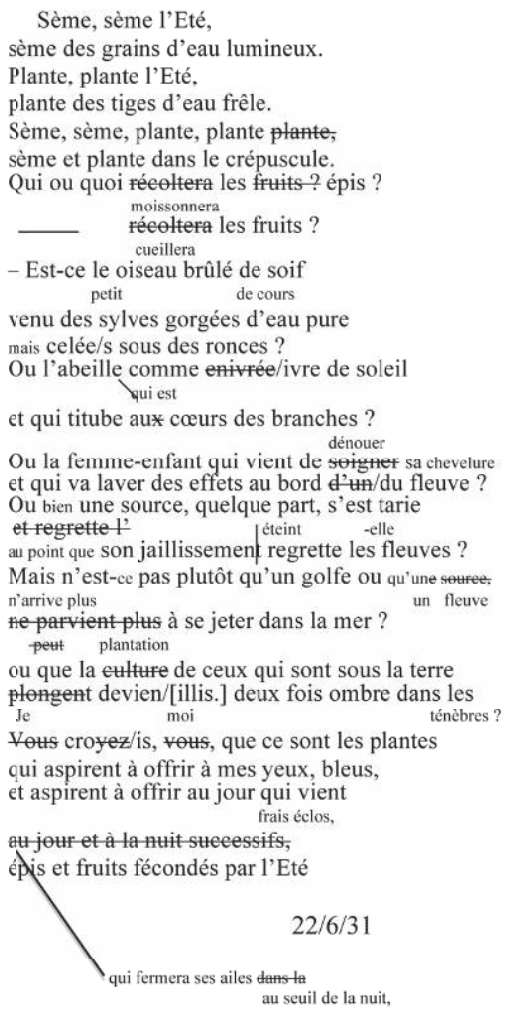 \\
\hline
\end{tabular}

Fig 3 : Transcription diplomatique des deux versions
ÉTÉ

1. Sème, sème l'été,

2. sème des grains d'eau lumineux.

3. Plante, plante l'été,

4. plante des tiges d'eau frêles

5. Sème, sème, plante, plante,

6. sème et plante dans le crépuscule.

7. Qui ou quoi moissonnera les épis ?

8. Qui ou quoi cueillera les fruits ?

9. Est-ce le petit oiseau brûlé de soif

10. venu des sylves gorgées de cours d'eau pure

11. celés, celés sous des ronces?

12. Ou l'abeille qui est comme ivre de soleil

13. et qui titube au cœur des branches ?

14. Ou la femme-enfant qui vient de dénouer sa chevelure

15. et qui a lavé des effets au bord du fleuve ?

16. Ou bien une source, quelque part, s'est-elle tarie

17. au point que son jaillissement éteint regrette les fleuves ?

18. Mais n'est-ce pas plutôt qu'un fleuve bruissant,

19. ici ou là, n'arrive plus jusqu'au golfe,

20. et n'arrive plus à grossir la mer ?

21. Ou que la plantation de ceux qui sont sous la terre

22. devient deux fois ombre dans les ténèbres ?

23. Je crois, moi, que ce sont les plantes

24. qui brûlent d'offrir à mes yeux parfois bleus

25. et brûlent d'offrir au jour frais éclos

26. qui fermera ses ailes au seuil de la nuit,

27. des épis et des fruits fécondés par l'été.

Fig 4 : Texte publié en 1934 (Éditions Henri Vidalie)

de contamination intertextuelle, n'est en tout cas pas décelable en malgache.

$\mathrm{Au}$ vers suivant, Rabearivelo échoue également à rendre les nuances latinisantes portées par l'adjectif «celés», dont le correspondant de la version malgache «miafina» (trouvé avec force peine comme en témoignent les nombreuses ratures) signifie seulement «caché».

Alors, certes, une part du sens se dérobe d'une langue à l'autre, et le poète travaille nécessairement avec deux palettes syntaxiques, deux nuanciers sémantiques, deux répertoires culturels différents. Mais il fait porter tout son effort sur un mouvement de rapprochement dont le cahier garde la trace.

\section{Un processus de contamination}

Le brouillon rédactionnel du poème montre clairement une interaction des deux systèmes linguistiques. Rabearivelo use fréquemment des effets poétiques de la contamination syntaxique. Ainsi, au vers 1 ( «Sème, sème l'été,») comme aux vers suivants, il faut comprendre le «Sème» de la version française comme un verbe conjugué à l'indicatif 


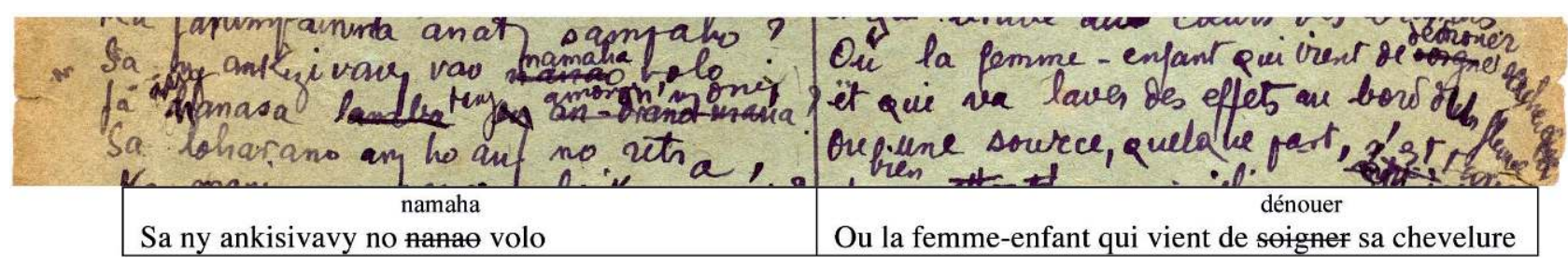

Fig. 6 : brouillon rédactionnel de Presque-Songes, $\mathrm{f}^{\circ} 1 \mathrm{v}$, vers 14 (détail)

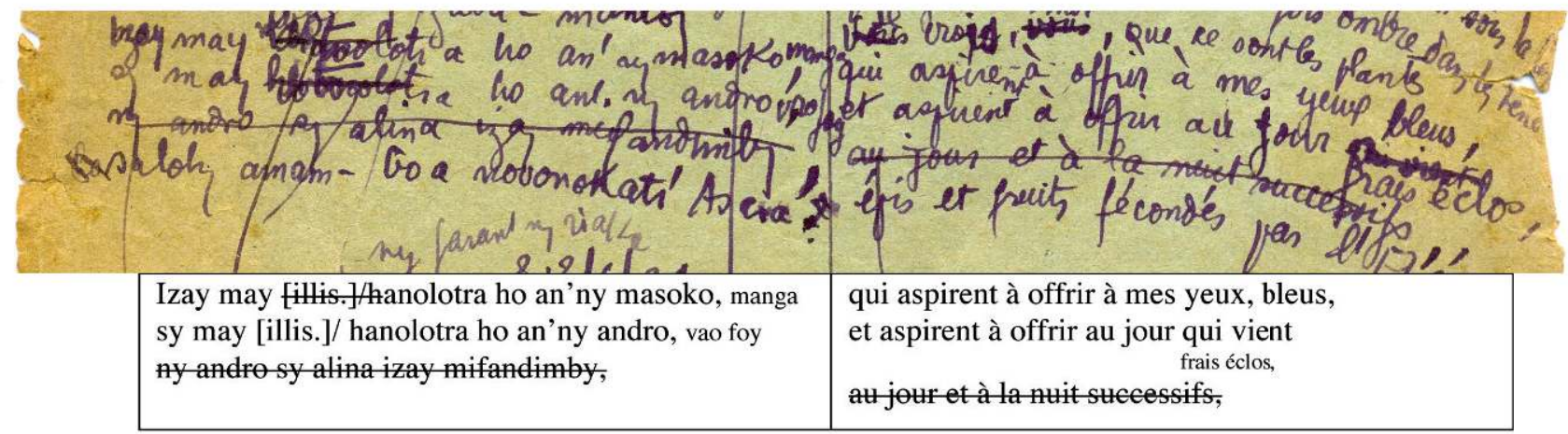

Fig 7 : brouillon rédactionnel de Presque-Songes, $\mathrm{f}^{\circ} 1 \mathrm{v}$, vers 23-25 (détail)

présent, construit selon un ordre syntaxique inversé VerbeSujet qui calque la syntaxe malgache.

Dans ce cas précis, l'influence de la syntaxe du malgache sur la construction de la phrase en français est particulièrement manifeste.

On observe également de nombreux exemples de contamination lexicale, dont un très beau spécimen au vers 2 avec le syntagme «grains d'eau», traduction littérale de la collocation «voan-drano » [goutte d'eau]. La littéralisation de l'expression en français confère au vers une puissance poétique singulière. Ce procédé est fréquent dans la suite du recueil; signalons simplement à titre d'exemple la traduction de l'expression «omby manga » par «bœuf bleu». Le poète joue en français de la polysémie que possède en malgache l'adjectif «manga», qui signifie «sauvage» quand il complète le substantif «omby» [bœuf] et qui, dans d'autres contextes, a le sens d' «excellent»... ou de «bleu»!

Mais il ne peut suffire, comme le souligne le théoricien Abdelkébir Khatibi, de pointer les exemples de contamination lexicale d'une langue dans l'autre :

[Le poème] s'écrit bel et bien par une pensée presque imperceptible et qui se renverse, transite dans l'impensé : le silence, l'intraduisible, le toujours-là-bas de l'innommé. De telles lois, qui s'élaborent selon la syntaxe du corps, ne peuvent être «plaquées» sur la surface du texte; car, elles sont le récit des langues qui parlent pour leur propre compte, le récit de la voix qui n'est plus infiniment séparée de l'écriture.
Les lois «presque imperceptibles» mentionnées par Khatibi peuvent être rendues visibles, décelables, par l'analyse génétique des mouvements de l'écriture. Au-delà des jeux ponctuels sur la traduction littérale d'un élément langagier, qu'il soit syntaxique ou lexical, il est manifeste que Rabearivelo a recherché un accord, quasi musical, entre les deux versions. Ces ajustements d'ordre lexical et syntaxique, rythmique et phonique, intervenant au stade de la première campagne de correction, vont dans le sens d'une plus grande précision, d'une plus grande justesse du vers dans les deux langues.

$\mathrm{Au}$ vers 14 par exemple, «nanao/soigner», biffé, est remplacé par «namaha/dénouer» (fig. 6).

Le verbe «manao» («nanao» au passé), très usuel, polysémique, est en effet doté d'un large spectre sémantique autour du sens principal de «faire». Il semble que le changement lexical ait été motivé par la nécessité de préciser le sens du vers malgache, dont la charge sémantique ou l'impact poétique devenait plus faible que sa traduction en français. Rabearivelo a donc opté pour une image plus concrète dans les deux langues.

On remarque la même exigence de précision imposée par le français dans les vers 23 à 25, dans lesquels la version malgache a été plusieurs fois retouchée (fig. 7).

L'adjectif «bleu» semble avoir été ajouté pour compléter le vers 23 de la version française resté en suspens; il est repris ensuite dans la version malgache où il est manifestement écrit en ajout («ny masoko, manga »). 

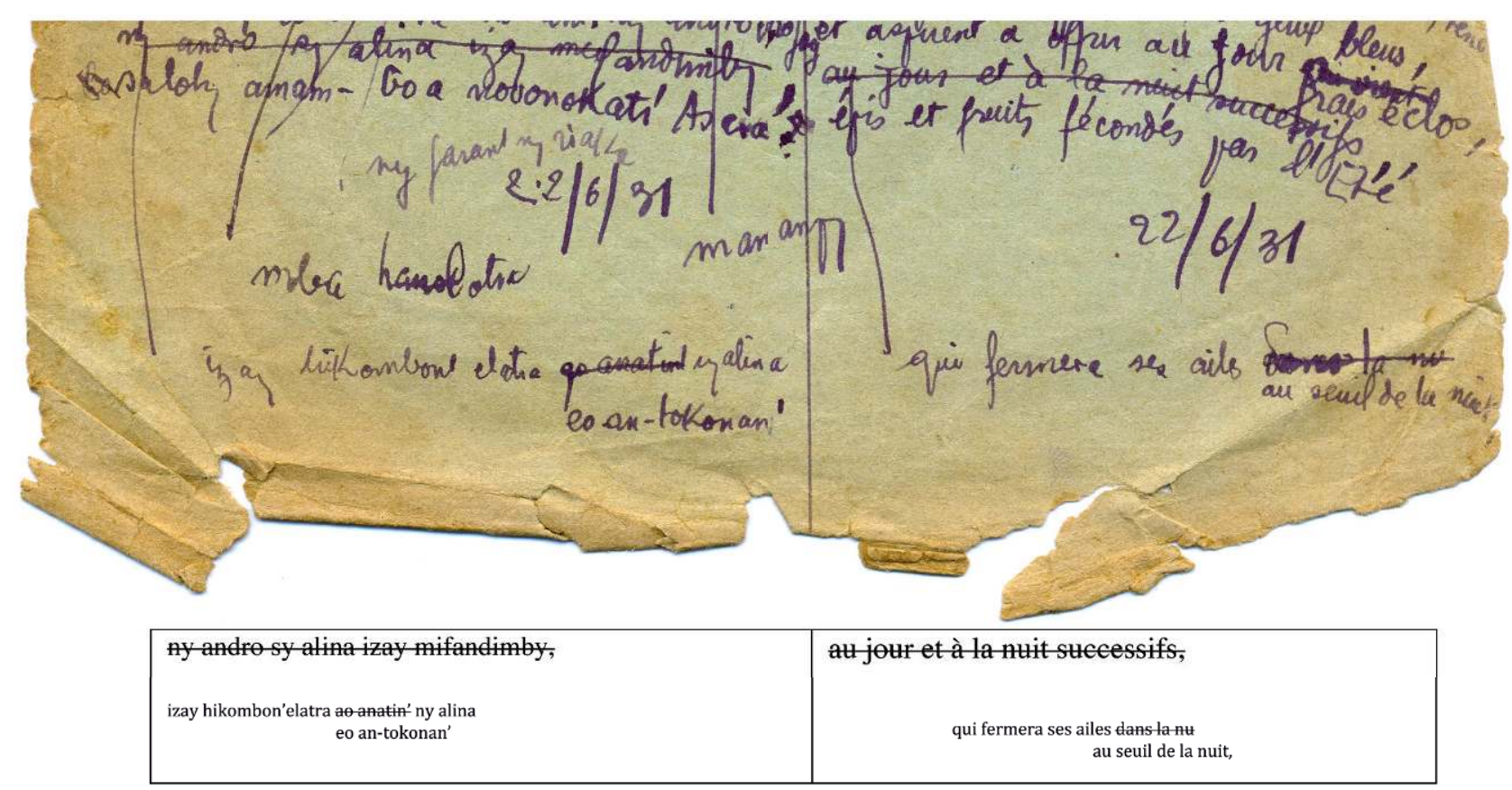

Fig. 8 : brouillon rédactionnel de Presque-Songes, $\mathrm{f}^{\circ}$ 1v, vers 25 (détail)

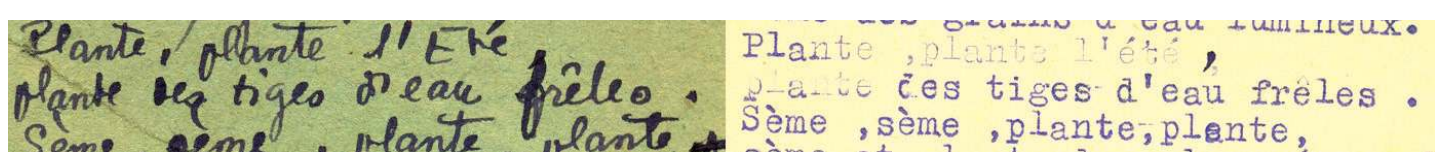

Fig. 9 : à gauche, brouillon rédactionnel de Presque-Songes, fo 1v, vers 3 et 4 ; à droite, dactylogramme du poème «Été» (détails)

Dans le vers suivant, la campagne d'écriture semble avoir débuté en malgache, puis le vers a été traduit en français. Le substantif «jour» a appelé un complément, «qui vient», dont l'équivalent le plus commun est rajouté en retour dans la version malgache («vao foy» [nouvellement éclos]). Cette formulation malgache, banale dans la langue mais très belle en traduction, provoque une nouvelle modification en français, Rabearivelo traduisant littéralement «vao foy » en français : «frais éclos».

Nous avons là un exemple manifeste de contamination entre les deux langues dans la fabrique du texte bilingue. Le même processus est décelable aux plans rythmique et phonique. Dans ce poème, il est lisible au vers 25 , entièrement retravaillé dans les deux versions (fig. 8). La première version était «ny andro sy alina izay mifandimby,» / «au jour et à la nuit successifs, ».

L'adjectif «successifs» traduisait littéralement «mifandimby ». Rabearivelo raye le vers entier pour le remplacer par : «izay hikombon'elatra ao anatin'ny alina» [qui fermera ses ailes dans la nuit]. Mais le complément de temps «ao anatin'ny alina» [dans la nuit] ne satisfait pas le poète, qui le supprime en lui substituant «eo an-tokonan'ny alina» / «au seuil de la nuit». Peut-on supposer que Rabearivelo cherchait à créer en français un rythme équivalent à celui du vers malgache où la fin du vers est mise en valeur par sa longueur, alors que «dans la nuit» tombait de façon plus abrupte?

L'interaction entre le français et le malgache que nous avons observée dans le premier brouillon manuscrit se poursuit dans la campagne de correction du folio dactylographié du poème (fig. 9).

Dans le vers 4, la formule de la version française, «plante des tiges d'eau frêles », subit une modification d'ordre syntaxique. Dans la version manuscrite, Rabearivelo accordait «frêle» avec «eau», choisissant ainsi de créer un hypallage (en français, puisqu'en malgache l'adjectif ne s'accorde jamais). L'accord se fait maintenant entre «frêles» et «tiges », dans un mouvement de normalisation linguistique qui tend à s'éloigner de l'influence du malgache.

Cependant, on y voit aussi la tendance inverse où le malgache vient bousculer le texte français, puisqu'aux vers 24 et 25 , le verbe «aspirent à» est modifié en «brûlent de», traduction littérale du malgache «may» [brûler] (fig. 10). 


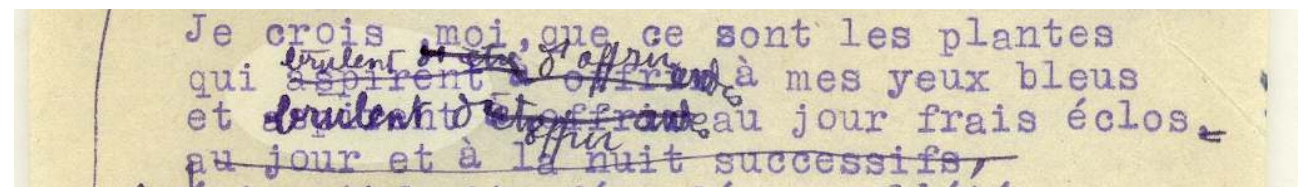

Fig. 10 : dactylogramme du poème «Été», vers 24-25 (détail)

Il semble que Rabearivelo ait toujours sous les yeux le brouillon rédactionnel bilingue, qui infléchit la campagne de relecture et de correction de la mise au net en français. L'avant-dernier vers du poème est ainsi modifié à la plume à la fois sur la dactylographie et sur le manuscrit, ce qui porte à croire que la modification a été faite conjointement sur les deux supports. Ce phénomène est également avéré pour les autres poèmes du recueil.

L'édition originale de 1934 est quasi identique à l'état dactylographié, excepté tout de même cet ajout sur épreuves de l'adverbe «parfois» au vers 24 («et brûlent d'offrir à mes yeux parfois bleus»), qui bouscule le sens. Mais si l'on se rappelle la polysémie de l'adjectif «manga» [bleu, excellent, sauvage] évoquée plus haut, on peut avancer que la version malgache travaille encore le poème, à un stade de l'élaboration où elle a physiquement disparu du papier.

\section{Conclusion}

Dans un des poèmes du recueil Presque-Songes intitulé justement «Le Poëme», Rabearivelo évoque le «chant intérieur» dont les mots se font l'écho :

[...] Paroles pour chant, dis-tu, paroles pour chant, paroles pour chant, pour désigner

le frêle écho du chant intérieur

qui s'amplifie et retentit,

tentant de charmer le silence du livre

et les landes de la mémoire,

ou les rives désertes des lèvres

et l'angoisse des cours 36 .

Traduire, transcrire ce chant intérieur, Rabearivelo y travaillera sans cesse dans sa poésie. Il l'évoquait déjà dans son poème «Per se» (Trèfles, 1923) : «Que résonne pure en ma musique / ta voix captive de l'indicible / ô chant vif jailli de mon âme ivre! 37 ». Dans Presque-Songes (1934) et Traduit de la nuit (1935), le poète continue de rêver cette langue intérieure. Ayant pris conscience très tôt de son aliénation linguistique, il l'interroge, tente des ouvertures poétiques, cherche des moyens de contourner le dilemme. Il le fera dans un premier temps en cherchant à «fiancer» ses deux langues poétiques, puis en tentant - et c'est le grand pari de Presque-Songes et Traduit de la nuit - une création conjointe, dont l'horizon est une quête de la langue non plus seulement «intérieure» mais «antérieure». Le grand rêve de Rabearivelo sera dès lors d'atteindre aux rivages de la «langue natale» (Baudelaire), de la langue suprême (Mallarmé38), d'une langue première : «cette voix intérieure aussi / que j'écoute depuis longtemps dans sa langue babélique ${ }^{39}$ ». Commentant son expérience de traducteur, il a souvent célébré cette quête de la langue idéale : «Je dirai le plaisir périlleux procuré par ce jeu qui fait courir à l'esprit même de la Poésie l'aventure la plus imprévue : celle de partir d'un même pays idéal pour l'inconnu de deux musiques différentes 40 ». Dans ses déclarations, Rabearivelo sacrifie donc largement à ce que Michel Beniamino nomme, dans une formule iconoclaste, la mythologie de l'anté-langue, en réclamant que les études critiques cessent d'enregistrer cette thèse comme vraie : "Les déclarations des écrivains à ce sujet sont le plus souvent enregistrées, faute d'être effectivement analysées, par certains ${ }^{41}$ ». À l'opposé des déclarations rétrospectives, l'approche génétique permet

36. « Le poème», Presque-Songes, op. cit., p. 517.

37. « Le live de ma poëtique», Trèfles, op. cit., p. 155.

38. Stéphane Mallarmé, Euvres complètes, Paris, Gallimard, coll. «Bibliothèque de la Pléiade», 1945, p. 363-364.

39. J. J. Rabearivelo, «Reconnaissance à Paul Gauguin» dans PresqueSonges, dans Euvres complètes, tome 2, op. cit., p. 583.

40. J. J. Rabearivelo, «D'un jeu plaisant mais périlleux », Capricorne $\mathrm{n}^{\circ} 3$, décembre 1930 .

41. Michel Beniamino, «Bilinguisme, intertextualité et récit identitaire», dans Robert Dion, Hans-Jürgen Lüsebrink et János Riesz (dir.), Écrire en langue étrangère, interférences de langues et de cultures dans le monde francophone, Québec/Francfort, Nota bene/IKO-Verlag, 2002, p. 320. 
d'analyser, selon le souhait de Beniamino, la réalité d'une création poétique bilingue en observant la contamination des langues en acte et la négociation permanente entre leurs irréductibilités foncières.

Dans les années 1980, Abdelkébir Khatibi théorise à partir de son itinéraire personnel entre arabe et français le concept de «bi-langue 42 », défini comme expérience de la séparation active entre les langues. «La bi-langue sépare, rythme la séparation, alors que toute unité est depuis toujours inhabitée 43 », écrit Khatibi. Évacuant la question de la langue originelle, il affirme que la bi-langue est une «scène du palimpseste et de la rature» :

Là s'inscrit l'entre-deux langues, la bi-langue. J'ai suggéré que la bi-langue est une double scription, une transcription, scène du simulacre, du palimpseste et de la rature. Dans la scène de la bi-langue, le simulacre ne renvoie pas intégrale- ment à la langue dite maternelle, il ne réduit pas la langue étrangère. Le simulacre : l'autre et du modèle et de la copie, l'autre du natal et de l'étranger44.

Cette direction de recherche, résumée ailleurs dans une formule programmatique : «de la bi-langue, dans ses effets de parole et d'écriture ${ }^{45} »$, peut entrer utilement en dialogue, au-delà du cas Rabearivelo, avec les études sur la genèse bilingue.

42. Le concept de «bi-langue» est élaboré par Abdelkébir Khatibi dans une lettre-préface à un ouvrage de Marc Gontard, repris et développé dans le récit Amour bilingue (Casablanca, Éditions Edif, 1992).

43. Abdelkébir Khatibi, Amour bilingue, op. cit., p. 109.

44. Abdelkébir Khatibi, «De la bi-langue», Écritures, 1982, p. 201.

45. Abdelkébir Khatibi (éd.), Du bilinguisme, Paris, Denoël, 1985, p. 11. 
ClaIRE RIFFARD, est ingénieure de recherche à l'ITEM (CNRS-ENS). Depuis 2008, elle y dirige l'équipe «Manuscrits francophones » qui étudie et édite les corpus littéraires majeurs d'Afrique et de la Caraïbe (Aimé Césaire, Mohammed Dib, Albert Memmi, Sony Labou Tansi, Ahmadou Kourouma, Jean-Joseph Rabearivelo, etc.). Elle a coédité l'œuvre complète de Jean-Joseph Rabearivelo (tomes I et II, CNRS Éditions, 2010-2012, 3000 p.) ainsi que Poèmes de Sony Labou Tansi (CNRS Éditions, 2015, 1272 p.).

claire.riffard@cnrs.fr

\section{Aperçus d'une genèse bilingue chez Jean-Joseph Rabearivelo}

Le poète malgache Jean-Joseph Rabearivelo (1903-1937) a légué à ses enfants le brouillon entièrement bilingue de son recueil poétique Presque-Songes, dont il n'a publié que la version française. La disposition des poèmes sur la page est toujours identique : version malgache à gauche, version française à droite, séparées par un trait central. Quelle version précède l'autre? Le brouillon du recueil atteste en réalité d'une écriture conjointe et d'une influence réciproque d'une version sur l'autre. L'article analyse en particulier la genèse du poème liminaire «Asara/Été», et montre un processus complexe où les deux versions du poème ne sont pas simplement successives mais interagissent dès la campagne d'écriture.

The Malagasy poet Jean-Joseph Rabearivelo (1903-1937) bequeathed to his children an entirely bilingual draft of his collection of poems Presque-Songes (Dream Images), of which he had published only a French version. The arrangement of the poems on the page remains identical: Malagasy version on the left, French one on the right, separated by a line in the middle. Which version precedes the other? In fact, the draft of this collection shows a joint writing and a reciprocal influence of one version on the other. This article analyzes in particular the genesis of the poem "Asara/Été", and shows the complex process in which the poem's two versions are not successive but interact in the different writing stages.

Der madagassische Dichter Jean-Joseph Rabearivelo (1903-1937) vermachte seinen Kindern den vollständig zweisprachigen Entwurf seiner poetischen Sammlung Presque-Songes, von der er nur die französische Version veröffentlicht hatte. Die Anordnung der Gedichte auf der Seite ist immer identisch: Madagassische Version auf der linken Seite, französische Version auf der rechten Seite, getrennt durch einen Strich in der Mitte. Welche Version geht der anderen voraus? Die Skizzen der Sammlung zeugen tatsächlich von einem simultanem Schreiben und einem wechselseitigen Einfluss einer Version auf die andere. Der Artikel analysiert insbesondere die Entstehung des Eröffnungsgedichtes „Asara/Été“ und zeigt einen komplexen Prozess, bei dem die beiden Versionen des Gedichts nicht einfach aufeinanderfolgen, sondern bereits in der ersten Phase des Schreibens interagieren.
E1 poeta malgache Jean-Joseph Rabearivelo (1903-1937) legó a sus hijos el borrador enteramente bilingüe de su libro poético Presque-Songes, del cual sólo publicó la versión francesa. La disposición de los poemas en la página es siempre idéntica: versión malgache a la izquierda, versión francesa a la derecha, separadas por una línea central. ¿Cuál de las dos versiones precede la otra? El borrador del libro revela en realidad una escritura conjunta y una influencia recíproca de una versión en la otra. Este artículo analiza en particular la génesis del poema liminar "Asara/ Été" y pone de manifiesto un proceso complejo en el que las dos versiones del poema no son simplemente sucesivas sino que interactúan desde la primera fase de la escritura.

O poeta malgaxe Jean-Joseph Rabearivelo (1903-1937) legou aos filhos o original bilingue da sua recolha de poemas Presque-Songes, de que apenas publicara a versão francesa. A disposição dos poemas na página é sempre a mesma: versão malgaxe à esquerda, francesa à direita, separadas por um traço central. Terá uma versão precedência sobre a outra? Na verdade, o original atesta que a escrita de ambas foi conjunta e que houve influências recíprocas. Analisando a génese do poema introdutório "Asara/Été", descobre-se um processo complexo, em que as duas versões do poema não estão simplesmente em sequência, mas interagem desde o início da escrita.

Il poeta malgascio Jean-Joseph Rabearivelo (1903-1937) ha lasciato in eredità ai suoi figli la bozza bilingue della sua raccolta Presque-Songes, di cui ha pubblicato solo la versione francese. La disposizione delle poesie sulla pagina è sempre identica : versione malgascia a sinistra, versione francese a destra, separate da una linea centrale. Quale versione precede 1'altra? La bozza attesta in realtà una scrittura congiunta e un'influenza reciproca di una versione sull'altra. L'articolo analizza in particolare la genesi della poesia che apre la raccolta, "Asara/Été", e mostra un processo complesso in cui le due versioni della poesia non sono semplicemente successive bensì interagiscono fin dalla prima fase di scrittura. 